\title{
Sarcoidosis in an Athlete
}

\author{
Laura Stefani* $^{1}$, MD; Ilaria Corsani ${ }^{1}$, MD; Paolo Manetti' ${ }^{2}$ MD; \\ Giulio Ciullini ${ }^{1}$, MD; Giorgio Galanti ${ }^{1}$, MD
}

\author{
Authors' Affiliation: \\ 1. Sports Medicine Center, \\ University of Florence, Italy \\ 2. ACF, Fiorentina Soccer Players \\ Team, Italy
}

* Corresponding Author:

Address: Viale Morgagni, 85 Careggi, Florence 50100, Italy

E-mail: laura.stefani@unifi.it

Received: Aug 31, 2010

Accepted: Dec 27, 2010

Key Words: Sarcoidosis; Athletes; Asthenia; Respiratory System; Granuloma; Treatment

\section{Abstract}

Background: Sarcoidosis is a multisystem granulomatous disorder of unknown etiology, characterized by "noncaseating granulomas" in different organs. Clinical signs are variable and dependent on the organ involved. Although it is often asymptomatic in athletes, considering the high level of athletic performance and the related risks due to the potential heart involvement, a particular diagnostic flowchart to consider some other diagnoses is required. The present case report aimed to focus on the clinical approach in case of a progressive weakness associated with a reduction in global performance of an athlete.

Case Presentation: Since October 2008 a 33-year-old Scandinavian professional soccer player has shown splitting headache, fever and impaired exercise tolerance. Despite some clinical aspects and symptoms that could address diagnosis of granulomatosis according to the current guidelines, the first hypothesis was indicative of a possible viral infection. Therefore, the athlete had received a drug-therapy resolving the headache and fever. However, because of the persisting weakness, several other clinical possibilities were evaluated following a more complete diagnostic flow-chart, blood and instrumental exams.

Conclusion: This case report focuses on the substantial absence of symptoms during the granulomatosis disease, which makes the differential diagnosis to be often complicating. Indeed, several additional exams are required in order to establish the presence of "Non-evolutive sarcoidosis- stage I", for which the therapy is not mandatory.

Asian Journal of Sports Medicine, Volume 2 (Number 1), March 2011, Pages: 57-62

\section{INTRODUCTION}

Sarcoidosis is a multisystem disease with unknown etiology, characterized by the presence of "noncaseating granulomas” in several organs. Despite the fact that exact prevalence of sarcoidosis is not known yet, its incidence in people like black Americans and Scandinavians is higher than others ${ }^{[1,2,3]}$. The greatest incidence has been observed in young adults aged between 20 and 40 years. Athletes 
can also be involved with an evident reduction in their sport performance. For almost $50 \%$ of the patients the diagnosis is often casual or occasional in case of the evident pulmonary radiographic abnormalities. Actually, the accurate etiology of the disease is not exactly known. It has been supposed to be mainly found in susceptible subjects as a consequence of an inflammatory response of organism to external inputs. The possible causes of immunologic response could be infections (viral, bacterial or mycobacterial) or inhalation of organic and inorganic agents. The inflammatory response is therefore characterized by accumulation of T-lymphocytes and macrophages with consequent organization of granulomas ${ }^{[4]}$. According to the current guidelines ${ }^{[4]}$, diagnosis of Sarcoidosis is actually based on the presence of typical symptoms (fever, fatigue, malaise, asthenia, dyspnea, cough, polyarthralgia, lymphadenopathy), high plasma level of a peculiar enzyme i.e. Angiotensin Converting Enzyme (ACE), presence of a bilateral hilar lymphadenopathy at the standard chest radiological exam and histological evidence of noncaseating epithelioid cell granulomas ${ }^{[4]}$. In order to confirm the diagnosis, some additional laboratory and instrumental exams (Purified Protein Derivative (PPD) skin test; serum level of erythrocyte sedimentation rate (ESR), C-reactive protein (PCR), Calcium, Phosphate; Kveim reaction test; complete microbiologic tests, Holter monitoring, echocardiography and thallium or gallium scans, magnetic resonance imaging (MRI), CT scans of the central nervous system and myocardial involvement can be helpful ${ }^{[4]}$. In spite of the fact that the most typical symptoms of sarcoidosis include cough, dyspnea, thoracic pain, fever and the weakness, a substantial absence of symptoms can be found in $90 \%$ of the patients, where bilateral hilar adenopathy and interstitial or alveolar opacities are often evident. Just in cases with big conglomerate clusters of granulomas, especially in the upper parts of lungs, due to the presence of the stenosis or bronchiectasis, the symptoms can be found ${ }^{[5]}$. Otherwise, involvement of different organs and tissues in athletes is frequent: in $10 \%$ of them the skeletal-muscles show acute polyarthritis, especially in hips. Besides, tendons and peritendinous tissue can be affected by a degenerative process that can result in breakage, especially in cases of protracted stress. The cardiac injury occurs more frequently in Japanese female population (approximately $20-47 \%$ of patients). This aspect determines $85 \%$ of deaths from sarcoidosis. However, the symptoms are observed only in $5 \%$ of cases and the survival up to five years is evident in about $60 \%$ of the subjects $^{[6]}$. A cardiac sarcoidosis characterized by wide fibrotic areas, can involve the pericardium, the endocardium, or the myocardium areas, mainly in the free wall of the left ventricle and in the interventricular septum creating some possible arrhythmias (from a first grade atrioventricular block to a complete block, atrial arrhythmias, sustained or non-sustained ventricular tachycardia) due to a reentry loop ${ }^{[7]}$. A potential evolution toward the left ventricle dilatation with heart failure has also been described $(8,9,10)$. Diagnosis of cardiac sarcoidosis is possible by the electrocardiographic, echocardiographic and radionuclide investigations (Gadolinium MRI exam), while the gallium-67 citrate (Ga) or Thallium-201 scintigraphy are normally used to assess the activity of the disease and the response to the corticosteroid therapy ${ }^{[11-13]}$. Cardiac biopsy is normally avoided because of the high level of risk associated with a low sensitivity ${ }^{[14]}$. In conclusion, the chest $\mathrm{XR}$ is generally the first exam to support the diagnosis of sarcoidosis. However, in case of doubt, a chest CT in addition to the bioptic sampling can be helpful to identify the granulomas and also to perform the differential diagnosis among other "granulomatous diseases". The respiratory functional tests remain however the main tests to estimate the disease's severity, showing normal values at the first stages and a restriction pattern with a reduced diffusion of $\mathrm{CO}$ in advanced stages ${ }^{[15]}$.

\section{CASE PRESENTATION}

The patient gave oral consent to participate in this case report. Since October 2008 a 33-years-old Scandinavian professional soccer player has shown splitting headache with fever and meningitic irritation signs like photophobia. 
The first diagnostic hypothesis was addressed toward a Toscana Virus infection that occurs frequently in this area. Initially, all serologic tests were negative. Head CT scans and magnetic resonance angiography (MRA) were negative either. Only a slight increase of ACE and unspecific flogosis-indexes like ESR and CRP were evident. Despite these results, he was submitted to a corticosteroid therapy with the resolution of the headache and fever. However, considering the persisting strong weakness with an inevitable negative impact on his sport performance, some other possible causes for the asthenia were considered and evaluated following an additional diagnostic flow-chart. The examinations made during one more month spent in the hospital showed normal values of the general blood tests, no serological signs of lymphoma along with a little increase of ACE plasma level. The serologic tests by polymerase chain reaction technique (PCR) including PPD skin test resulted to be negative except a past Toscana Virus (IgG) infection.

Among the cardiological investigations, the echocardiographic exam was normal while Maximal Ergometric Test (MET) showed sporadic singular monomorphic ventricular extrasystoles. In addition, 24hour Holter-monitoring ECG showed some polymorphic ventricular extrasystoles, partially organized in short bigeminal rhythm, and monomorphic and polymorphic couples at rest condition.

The respiratory functional tests described a pattern compatible with a slight obstruction and a normal CO diffusion. The chest XR was characterized by a moderate parenchymal involvement due to a hilar lymphadenopathy (Fig. 1). The abdominal echography showed an enhancement of the liver and spleen dimensions at the presence of abdominal lymph nodes, presence of which was confirmed by the abdominal and chest CT (Fig. 2). Increase of inguinal, mediastinal, supraclavicular and cervical lymph nodes with the same pattern were also described (Fig. 3). The additional evaluation by Positron Emission Tomography (PET/CT) showed a mediastinal and supraclavicular areas with higher glucose-metabolism than others. In the meantime, the athlete was quite asymptomatic and the weakness was the sign more constant. Therefore, diagnosis of sarcoidosis was supposed. The athlete was submitted to a bioptic
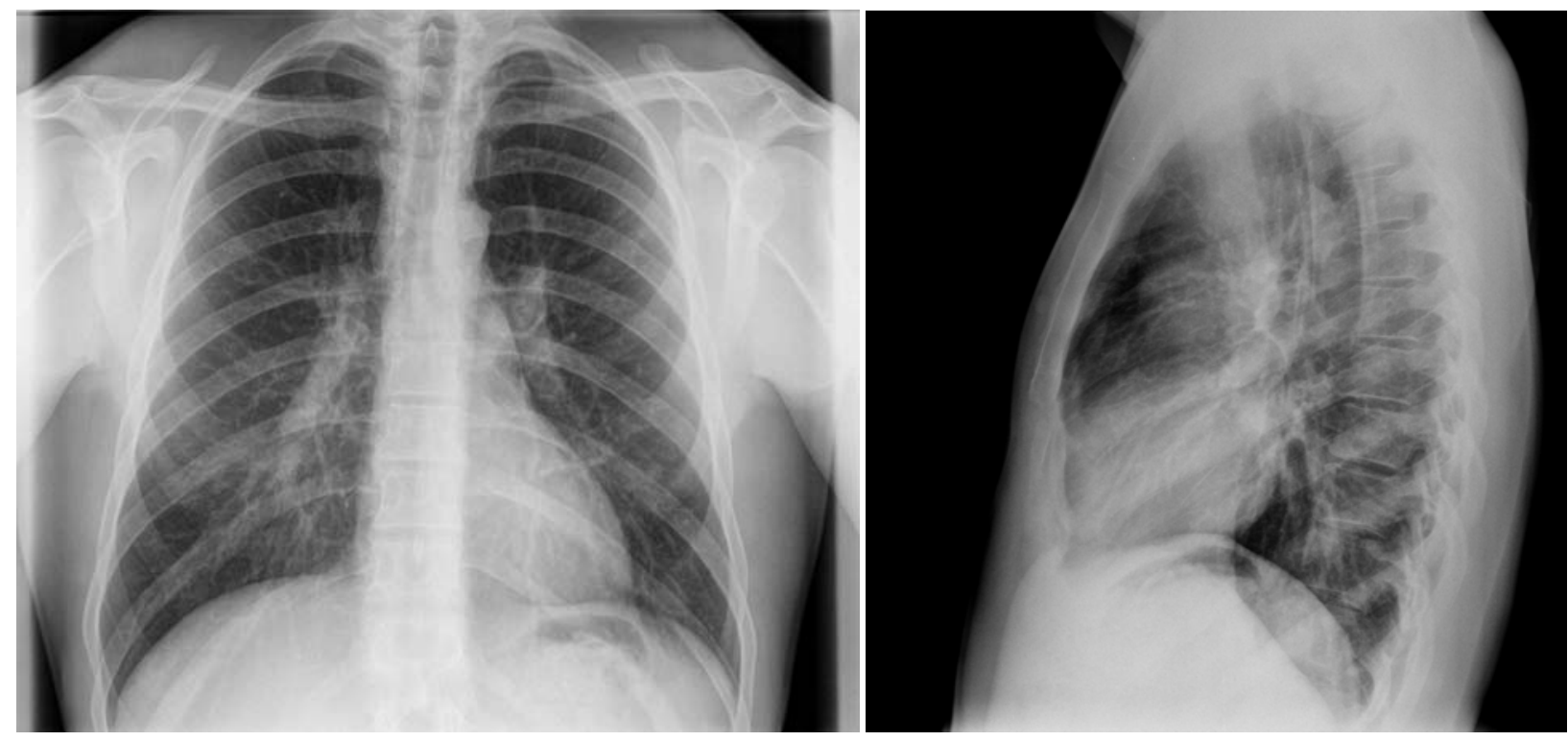

Fig. 1: The pictures of the chest by X-ray exam in two different visualizations show the pulmonary involvement due to a hilar lymphadenopathy 


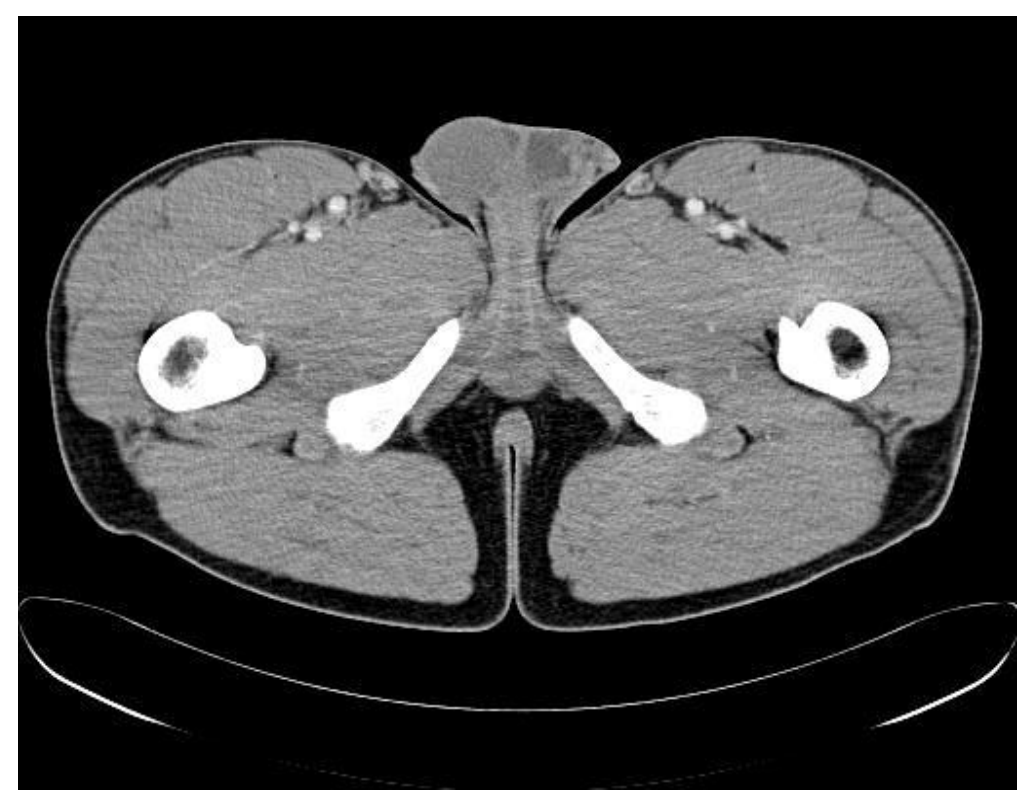

Fig. 2: The CT image shows the presence of inguinal lymph nodes

sampling of the laterocervical lymph nodes confirming the diagnosis previously hypothesized. Biopsy showed “noncaseating granulomas".

Considering the quite good clinical condition of the athlete, no more additional pharmacologic therapy except one month of detraining was advised. He was in a fairly good physical state thirty days later, and the follow-up exams didn't show any more progression of the disease. He gradually restarted his training after four months and he actually was in good performance.

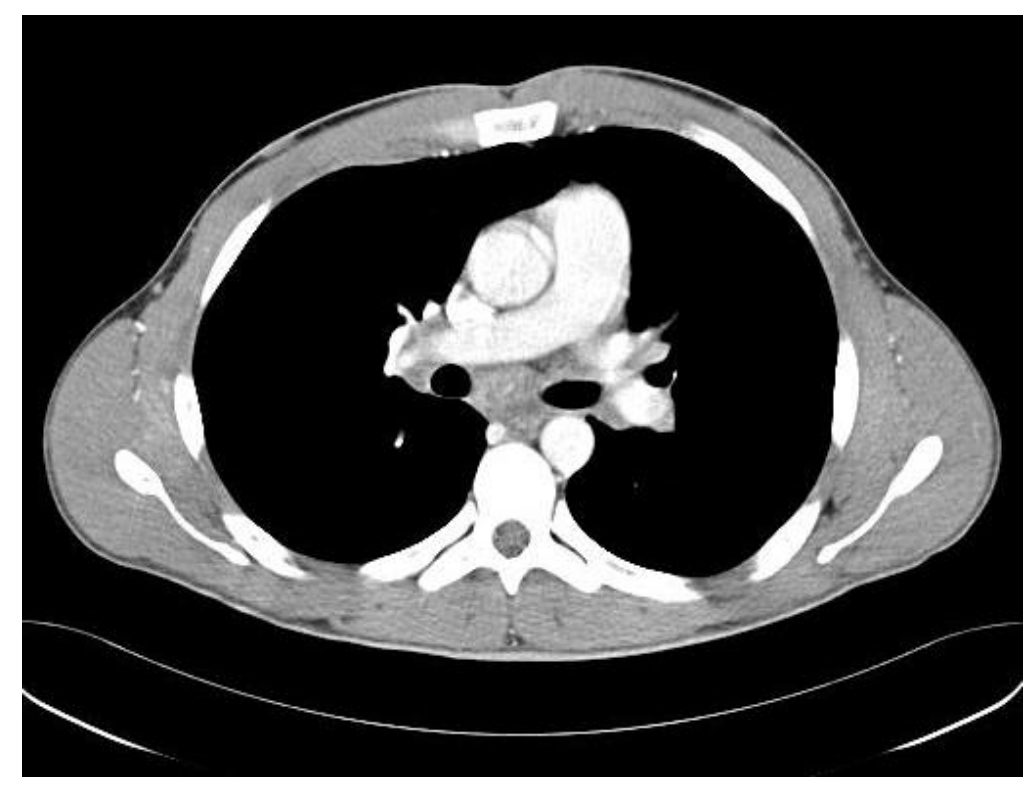

Fig. 3: The CT image shows the increase of mediastinal lymph nodes 


\section{DISCUSSION}

The symptom "asthenia" in a professional athlete is often described as loss of his global performance. In case of "unspecific symptoms" like headache, muscular fatigue and fever, it is necessary to reduce the time of sportive seasonal training to avoid the negative consequences of the overtraining. When it is possible to exclude any relation between symptoms and sport activity, it is correct to suspect a common pathology as flu syndrome or infective pathologies. Otherwise, according to previous examples of cases reported ${ }^{[22,23]}$, where exclusively unspecific clinical symptoms are evident and the most common pathologies can be excluded, some other diseases as lymphoma, tuberculosis, rheumatoid arthritis, rheumatic fever and mycosis have to be considered. Besides, when these ones can be excluded, it is consequently reasonable to think of sarcoidosis. Fatigue is the most common and the major problem in young patients with sarcoidosis, submitted or not submitted to a corticosteroid therapy. As no direct relation is often evident between fatigue and clinical parameters, it is very difficult to evaluate this aspect. No standard quantification of these symptoms has been determined yet. The Fatigue Assessment Scale (FAS), and the 6-minute walk ${ }^{[15-17]}$ can be considered now as the exclusive valid instruments to assess this one and are able to show the impaired exercise tolerance due to multiple factors above ${ }^{[18-20]}$. Sarcoidosis can often involve unexpected $\operatorname{organs}^{[22,23]}$ and only the evidence of noncaseating granulomas can confirm the doubt.

Correct diagnosis of sarcoidosis is therefore possible following a flow-chart to obtain a complete differential diagnosis panel, considering the presence of symptoms, the radiologic signs, in addition to a histopathologic confirmation. After the anamnesis and the physical examination several other exams are necessary:

- $\quad$ Complete blood tests

- ECG, echocardiogram, ECG sec. Holter

- Respiratory functional tests

- Chest XR

- Chest CT: especially in advanced stages of disease, when the radiologic images can simulate pulmonary metastasis
- MRI or scintigraphy with Gallium-67 or Tallium-201

- Transbronchial bronchoscopy and biopsy

Ninety percent of patients have a spontaneous resolution after two years, while restarting the disease is evident in less than $10 \%$ of them. On the contrary, sarcoidosis becomes chronic with permanent issues in $30 \%$ of the cases. The symptomatic subjects are normally submitted to a corticosteroid therapy (Prednisone $0.3-1 \mathrm{mg} / \mathrm{Kg} /$ day until a maximum of 40 $\mathrm{mg} /$ day). If the patient shows a good resolution of the disease within the first two weeks of therapy, he can be considered a "responder". When the corticosteroid therapy is interrupted, restart of the disease activity is possible.

In cases of side-effects or if the athlete is "nonresponder", an alternative therapy can be tried with azathioprine, cyclophosphamide, chlorambucil, chloroquine, pentoxifylline or infliximab ${ }^{[21,14]}$. The rate of the follow-up depends on stage and degree of the disease: every 4-8 weeks in subjects with acute sarcoidosis and corticosteroid therapy can be sufficient, whereas a 3-4-month period is acceptable in the first years and more rarely afterwards if the disease is indolent ${ }^{[21]}$.

\section{CONCLUSION}

The data obtained show a "sarcoidosis on first stage", which has not been developed yet and is exclusively characterized by clinical manifestations, i.e. the persistent asthenia.

This case report supports the assumption that therapy is not normally necessary in asymptomatic subjects. Respiratory functional tests and blood markers of disease are often sufficient, especially when the symptoms are restricted to a weakness. A different period of detraining is, in fact, sufficient to restitute the performance previously lost.

Conflict of interests: We declare that no founds have been received for this work. 


\section{REFERENCES}

1. Fernandez- Fabrellas E. Arch Bronconeumol 2007;43:92-100.

2. Rybicki BA, Mayor M, Popovich J, et al. Racial differences in sarcoidosis incidence: a 5-year study in a health maintenance organization". Am J Epidemiol 1998;148:100-1.

3. Westney GE, Judson MA. Racial and ethnic disparities in sarcoidosis: from genetics to socioeconomics. Clin Chest Med 2006;27:453-62.

4. Hunninghake GW, Costabel U, Rizzato G, Semenzato G. Statement on sarcoidosis. Am J Respir Crit Care Med 2005; 160:736-55.

5. Kinder BW, Shariat C, Collard HR, et al. Undifferentiated connective tissue disease-associated interstitial lung disease: changes in lung function. Lung 2010;188:143-9.

6. Smedema JP, Snoep G, van Kroonenburgh MP, et al. Cardiac involvement in patients with pulmonary sarcoidosis assessed at two university medical centers in the Netherlands. Chest 2005;128:30-5.

7. Riezzo I, Ventura F, D’Errico S, et al. Arrhytmogenesis and diagnosis of cardiac sarcoidosis. An immunohistochemical study in a sudden cardiac death. Forensis Sci Int 2009;183:1-5.

8. Barton JH, Tavora F, Farb A, et al. Unusual cardiovascular manifestation of sarcoidosis, a report of three cases: coronary artery aneurysm with myocardial infarction, symptomatic mitral valvular disease, and sudden death from ruptured splenic artery. Cardivasc Pathol 2009;19:119-23.

9. Kim JS, Judson MA, Donnino R, et al. Cardiac sarcoidosis. Am Heart J 2009;157:9-21.

10. Kollerman J, Roos G, Helpap B. Sudden cardiac death from unrecognized cardiac sarcoidosis. Pathologe. 2001;22:141-4.

11. Manins V, Habersberger J, Pfluger $\mathrm{H}$, et al. Cardiac magnetic resonance imaging in the evaluation of cardiac sarcoidosis: an Australian single-centre experience. Intern Med J 2009;39:77-82.

12. Pierre-Louis B, Prasad A, Frishman WH. Cardiac manifestation of sarcoidosis and therapeutic options. Cardiol Rev 2009;17:153-8.

13. Tolat AV, Norris RB, Wong KL, et al. Cardiac arrest in an athlete as the initial clinical presentation of cardiac sarcoid. Heart Rhythm 2006;3:88-90.

14. Tandri H, Bomma C, Calkins H. Unusual presentation of cardiac sarcoidosis. Congest Heart fail. 2007;13:116-18.

15. Medinger AE, Khouri S, Rohatgi PK. Sarcoidosis: the value of exercise testing. Chest 2001;120:93-101.

16. De Vries J, Michielsen H, Van Heck GL, Drent M. Measuring fatigue in sarcoidosis: the Fatigue Assessment Scale (FAS). Br J Health Psychol 2004;9:279-91.

17. De Vries J, Rothkrantz-Kos S, van Dieijen-Visser MP, Drent M. The relationship between fatigue and clinical parameters in pulmonary sarcoidosis. Sarcoidosis Vasc Diffuse Lung Dis 2004;21:127-36.

18. Baughman RP, Sparkman BK, Lower EE. Six-minute walk test and health status assessment in sarcoidosis. Chest 2007;132:207-13.

19. Kabitz HJ, Lang F, Walterspacher S, et al. Impact of impaired inspiratory muscle strength on dyspnea and walking capacity in sarcoidosis. Chest 2006;130:1496-502.

20. Cohen NP, Gosset J, Staron RB, Levine WN. Vertebral sarcoidosis of the spine in a football player. Am J Orthop 2001;30:875-7.

21. Lower EE, Harman S, Baughman RS. Double-blind, randomized trial of Dexmethylphenidate Hydrochloride for the treatment of sarcoidosis-associated fatigue. Chest 2008;133:1189-95.

22. Miller AC, Chacko T, Rashid RM, Ledford DK. Fever of unknown origin and isolated non caseating granuloma of the marrow: could this be sarcoidosis?. Allergy Asthma Proc 2007;28:230-5.

23. Moret Ch, Meier P, Rotman S, et al. Fever of unknown origin with granulomatous hepatitis, uveitis and acute renal failure. Praxis (Bern 1994) 2005;94:25-30. 